\title{
Produção Científica Sobre Avaliação da Inteligência: O Estado da Arte
}

\author{
Carolina Rosa Campos* \& Tatiana de Cássia Nakano \\ Pontifícia Universidade Católica de Campinas, Campinas, Brasil
}

\begin{abstract}
RESUMO
O presente estudo, do tipo estado da arte, teve como objetivo identificar aspectos relevantes da produção científica brasileira sobre avaliação da inteligência no período de 2000 a 2010 em quatro bases de dados eletrônicas (Scielo, Pepsic, Psicinfo e Capes). Um total de 263 trabalhos foram analisados cujos resultados demonstraram um crescimento do estudo da temática, principalmente entre os anos de 2005 a 2007, com tendência a trabalhos do tipo empírico (71,5\%), baseados no modelo psicométrico $(51,71 \%)$, fazendo uso de oito diferentes instrumentais, com destaque para a BPR-5 $(26,6 \%)$, WISC, MSCEIT e RAVEN (representando 19,3\% cada), predominantemente em amostras envolvendo jovens $(27,8 \%)$. Almeja-se que este trabalho inspire outros pesquisadores, contribuindo para a expansão do conhecimento acerca da inteligência na população brasileira.
\end{abstract}

Palavras-chave: avaliação da inteligência; avaliação psicológica; estado da arte.

\section{ABSTRACT \\ Scientific Production in Intelligence Assessment: The State of Art}

The present state of the art study aimed to identify relevant aspects of Brazilian scientific production on the assessment of intelligence, in the period of 2000 to 2010 in four electronic databases (Scielo, Pepsic, Psycinfo and Capes). A total of 263 papers were analyzed. Results showed a growth of interest on the study of this issue, especially between the years 2005 to 2007 , with a tendency to empirical work $(71.5 \%)$, based on psychometric model $(51.71 \%)$, using eight different instruments, especially BPR-5 (26.6\%), WISC, Raven and MSCEIT (representing 19.3\% each), predominantly in samples involving young people $(27.8 \%)$. The authors hope that this work will inspire other researchers, contributing to the expansion of knowledge about intelligence in the Brazilian population.

Keywords: evaluation of intelligence; psychological assessment; state of the art.

Dentre os construtos individuais que têm sido investigados nos últimos 100 anos, aqueles relacionados à inteligência, habilidade, aptidão e realização têm ganhado destaque (Wilhelm \& Engle, 2005). Neste período, dois movimentos podem ser relatados. Um primeiro, focado na avaliação e previsões práticas das diferenças individuais para o sucesso acadêmico e ocupacional, e um segundo, composto por uma visão mais reducionista, voltada à procura de marcadores psicológicos e psicofísicos que poderiam indicar o potencial intelectual ou capacidade do indivíduo (Ackerman \& Beier, 2005).
Devido aos benefícios resultantes desse tipo de investigação, tem sido notado um aumento no interesse pelo estudo da inteligência e suas medidas, "especialmente porque elas são frequentemente relacionadas a comportamentos socialmente valorizados, tais como desempenho acadêmico, desenvolvimento profissional, traços de personalidade, entre outros" (Santos, Noronha \& Sisto, 2005, p. 192). Devido a esta valorização, o construto tem sido foco de discussão entre os estudiosos da inteligência, diante principalmente das divergências entre as teorias que foram desenvolvidas, à falta de consenso entre as várias definições existentes, e também sobre a maneira de avaliá-lo (Roazzi \& Souza, 2002). Isso porque a inteligência não é uma

* Endereço para correspondência: Carolina Rosa Campos - carolene_crc@ hotmail.com 
preocupação do presente, havendo esboços das definições e das controvérsias atuais ao longo de mais de um século de estudo (Almeida, Guisande \& Ferreira, 2009).

Uma rápida retomada histórica, com o objetivo de apontar os modelos e autores de maior destaque na investigação da inteligência, mostra que estudos envolvendo a medição dessa capacidade tiveram início em 1882 com a tentativa de Galton de medir as diferenças individuais por meio de habilidades como discriminação visual e auditiva, tempo de reação e força (Brody, 2000). Também nessa época, por volta de 1900, Binet dá início à avaliação de crianças que, por motivos de retardo mental ou atrasos desenvolvimentais, necessitavam de educação especial, realizadas por meio de uma variedade de medidas físicas e fisiológicas, bem como testes baseados na investigação de processos mentais (Urbina, 2004). Ainda de acordo com a autora, os resultados desses estudos acabam levando-o, juntamente com um colaborador, à criação de um teste de inteligência (Binet-Simon Scale), o qual acabou sendo rapidamente traduzida para outras línguas e adaptada para uso fora da França, onde foi criada.

Uma revisão posterior da escala (Stanford-Binet Intelligence Scale) dá origem ao conceito de Quoeficiente de Inteligência (Q.I.), um escore que representaria o nível de habilidade mental em diferentes idades, proveniente da divisão entre a idade cronológica e a idade mental. Embora amplamente divulgado, o modelo passa a ser questionado, primeiramente por Thurstone em 1938, a partir da formulação de uma proposta de sete aptidões diferenciadas (compreensão verbal, fluência verbal, aptidão numérica, velocidade perspectiva, aptidão espacial, memória e raciocínio) e posteriormente por Guilford em 1959, a partir da criação do modelo Structure-of-Intellect, modelo no qual previa a inteligência a partir da combinação das tarefas mentais, dos conteúdos das tarefas e produtos possíveis do trabalho mental (Almeida \& Primi, 2009), de modo que, nesse modelo, as habilidades independentes se organizariam em três níveis: 150 habilidades consideradas fator de primeira ordem poderiam se combinar de modo a originar 85 fatores de segunda ordem, os quais, por sua vez, formariam 16 fatores de terceira ordem (Brody, 2000). Com essa visão, ambos modelos dão origem à ideia de que as habilidades cognitivas poderiam organizar-se de acordo com modelos hierárquicos, de modo que tais autores passam a questionar a ideia de uma visão unitária da inteligência, defendendo que esta seria composta por aptidões independentes.
A partir desse momento, o principal ponto de controvérsia e tensão entre as teorias de inteligência passa a referir-se às discussões acerca dos fatores que a comporiam, iniciadas por Spearman. Partindo do reconhecimento da possibilidade de existência de erros de medida, o autor desenvolve o conceito de inteligência geral. Desde então, alguns modelos passam a argumentar sobre a existência de uma única habilidade geral (fator g) que representaria toda a atividade intelectual, ao passo que outros modelos começam a defender a inexistência de um fator geral e, no lugar disso, um conjunto de habilidades básicas ou primárias (Primi e cols, 2001). Dessa maneira, a principal questão presente na primeira metade do século passado, era voltada aos estudos fatoriais da inteligência, acerca da estrutura (quantas) e definição (quais) das capacidades intelectuais (Primi, 2003). Por esse motivo, ainda segundo o autor, a grande maioria dos estudos psicométricos da inteligência, desenvolvidos nas primeiras sete décadas do século $\mathrm{XX}$, tiveram como foco a aplicação de baterias de testes e posterior emprego da análise fatorial, visando descobrir como esses testes se correlacionavam de modo a definir, a partir dos agrupamentos formados, as estruturas da inteligência (em termos de fatores, habilidades e aptidões).

Nesse panorama, destaque deve ser dado ao surgimento da teoria proposta por Cattell, segundo o qual, a inteligência teria dois componentes diferentes: a inteligência fluida (Gf) e a inteligência cristalizada (Gc). Nela, o primeiro tipo seria compreendido como a capacidade do indivíduo de realizar operações mentais frente a uma tarefa nova e que não podem ser executadas automaticamente, estando associada a componentes pouco dependentes de conhecimento prévio e influência cultural, dependendo muito mais de fatores biológicos e genéticos (Almeida, Lemos, Guisande \& Primi, 2008; Flanagan, McGrew \& Ortiz, 2000). Por outro lado, o segundo seria desenvolvido a partir de experiências culturais e educacionais, presente na maioria das atividades escolares, de modo a representar diferentes tipos de capacidades exigidas na solução de problemas cotidianos, podendo ser entendida como uma forma de inteligência social ou de senso comum, no qual é necessário o investimento na aprendizagem (David \& Downing, 2000; Schellini, 2006). Esse conceito de inteligência fluida e cristalizada é assumido posteriormente por Horn e Carroll, sendo revisado e complementado a partir da incorporação de nove habilidades específicas: inteligência fluida e cristalizada, visual, auditiva, recuperação a curto prazo, recuperação a longo prazo, velocidade de processamento e de 
decisão, bem como conhecimento quantitativo (Horn $\&$ Noll, 1997), com a inclusão posterior de outra habilidade: comportamentos relacionados à leitura e escrita (McGrew, 1997). Tais habilidades estariam compreendidas dentro de um conceito de três estratos, os quais representariam diferentes níveis de habilidades (Carroll, 1997).

Sem dúvida, um dos grandes avanços na área foi conseguido mais recentemente com o surgimento da teoria CHC (Cattell-Horn-Carrol), enquanto proposta de definição e avaliação da inteligência (Almeida, Primi \& Ferreira, 2008) resultante da junção dos três modelos. Segundo os autores, a popularidade crescente dessa teoria e o fato de algum consenso ter sido obtido entre os pesquisadores, tem originado um movimento dos autores de provas de inteligência à procura de uma maior adequação de suas baterias a este modelo teórico, dada sua abrangência, na tentativa de aumentar a validade preditiva dos instrumentos nas múltiplas situações de prática profissional em que essa avaliação se fizer necessária. Assim, a tendência notada tem sido a de reanálise dos componentes medidos nas baterias já existentes (Chiodi \& Wechsler, 2009).

A teoria envolve a ideia de camadas dispostas em três níveis, sendo o Estrato I composto por 65 fatores específicos, os quais encontrariam-se ligados ao formato dos problemas cognitivos propostos pelos testes psicométricos, o Estrato II que agruparia estes fatores específicos em oito fatores amplos (raciocínio, conhecimento-linguagem, memória-aprendizagem, percepção visual, percepção auditiva, produção de ideias, velocidade de processamento cognitivo e velocidade de decisão) e o Estrato III, que corresponderia ao fator $g$, de forma a indicar a existência de operações cognitivas comuns a todas as atividades mentais (Primi, 2002).

Também bastante divulgados atualmente, podem ser citados os estudos de Gardner (1995) e sua teoria das inteligências múltiplas. Nela, uma ampla gama de capacidades foram agrupadas em sete categorias abrangentes: inteligência linguística, lógico-matemática, espacial, interpessoal, intrapessoal, musical e corporal (Armstrong, 2003), sendo as três primeiras relacionadas a habilidades medidas por testes convencionais de inteligência, ao passo que as demais não (Davidson \& Downing, 2000). Ainda de acordo com os autores, Gardner acreditava que as inteligências eram relativamente independentes uma das outras, ainda que, em determinadas situações, pudessem ser necessárias conjuntamente. Segundo Chen e Gardner (1997), reconhece-se, no entanto, a existência de um número maior de inteligências e subinteligências, ainda que essas sete caracterizem mais adequadamente o intelecto humano, reforçando a ideia de independência entre elas.

Assim, o que se pode verificar, é que, ao longo do último século, evidências acumuladas permitiram clarificar a concepção de multidimensionalidade da inteligência humana, ainda que, ao longo desse período, as teorias de inteligência diferiram em relação ao número de habilidades relevantes envolvidas nesse construto e sua organização (Schulze, 2005). Entretanto, não se pode negar que um considerável corpo de conhecimento foi construído através do método psicométrico de avaliação da inteligência (Heck e cols., 2009), principalmente nas últimas décadas. Importantes avanços também foram obtidos a partir da incorporação e uso de novas técnicas estatísticas, as quais têm possibilitado estudos mais rigorosos acerca das estruturas hipotéticas assumidas pelos testes de inteligência, incluindo a análise fatorial confirmatória, teoria de resposta ao item, testagem adaptativa e computadorizada e modelos baseados em evidências (Ittenbach, Esters \& Wainer, 1997; Roberts e cols., 2005; Thorndike, 1997). Tais avanços fizeram com que a tendência atual na área, segundo opinião de Roberts, Markham, Matthews e Zeidner (2005), esteja marcada pela necessidade de mudança nos procedimentos utilizados no desenvolvimento de testes, na natureza das tarefas medidas, nos aspectos de habilidade que são avaliados e nos tipos de interpretações que vêm sendo feitas a partir dos escores obtidos nos testes, de maneira a tentar diminuir a distância, muitas vezes existente, entre a teoria e a prática, suprindo a ausência de instrumentos mais sofisticados e com propósitos práticos.

Dada a relevância do consrtruto, o interesse em sua medida também se fez muito presente na literatura, de forma que os testes de inteligência vêm sendo utilizados na avaliação psicológica com o objetivo de atender a uma grande variedade de objetivos e contextos, constantemente em expansão. Dentre suas áreas de utilização na Psicologia, Alves (1998) e Sisto, Ferreira e Matos (2006) destacam a área ocupacional, seleção e orientação profissional, a orientação de carreira, a área educacional para a avaliação e acompanhamento de crianças com problemas de aprendizagem, com deficiência intelectual ou superdotadas, a área clínica para realização de diagnósticos, a área hospitalar e a área judiciária, de modo que os testes continuam como ponto principal de referência para muitas decisões relacionadas à área educacional assim como psicológica. Também Roberts e cols. (2005) salientam que o 
estudo da inteligência tem sido, cada vez mais, valorizado em outros diversos e diferentes domínios, incluindo economia, marketing, medicina e genética. Contraditoriamente, mesmo diante desse leque de possibilidades de uso, o que se nota, no Brasil, é que, embora venham sendo realizados há aproximadamente cem anos, os estudos sobre inteligência ainda são poucos se comparados com o cenário internacional (Sisto, Santos \& Noronha, 2007).

Dessa forma, considerando a diversidade de modelos teóricos explicativos da inteligência, sua importância histórica para a Psicologia e a diversidade de instrumentais que vêm sendo utilizados, nacionalmente e internacionalmente, na avaliação desse construto, o presente estudo tem por objetivo constituir-se em um trabalho do tipo estado da arte. Visa pesquisar e identificar aspectos relevantes da produção científica brasileira sobre avaliação da inteligência, na tentativa de mapear e discutir o que vem sendo publicado na literatura científica nacional no período compreendido entre os anos 2000 e 2010.

A importância desse tipo de estudo ampara-se na possibilidade de clarificação dos enfoques que têm sido dados nos estudos, o conhecimento do tipo de investigação que vem sendo privilegiada, bem como a qualidade e efetividade dessas investigações (Witter, 1999). Outras vantagens são apontadas por Méis e Letas (1996) ao afirmarem que conhecer o atual estado de produção científica sobre determinado tema permite a identificação de lacunas que necessitam de maiores investigações, a caracterização de instrumentos mais utilizados e o conhecimento dos conceitoschave que vêm permeando as investigações científicas, de forma que trabalhos sobre produção científica são de grande relevância, pois fornecem um mapeamento das contribuições, necessidades e déficits da produção em uma determinada área (Lustoza, Oliveira \& Mello, 2010).

\section{MÉTODO}

\section{Material}

$\mathrm{Na}$ intenção de descrever e analisar a literatura científica sobre a temática, foi realizada uma busca em quatro bancos de dados, sendo três de periódicos científicos: SCIELO (www.scielo.br), PEPSIC (www.pepsic. bvsalud.org) e PsycInfo (www.apa.org/pubs/databases/ psycinfo/index.aspx) e uma base de teses e dissertações: CAPES (www.capes.gov.br), em um total de 263 trabalhos.

\section{Procedimento}

A partir da busca pela palavra-chave "inteligência", limitada ao período de 2000 a 2010, foram encontrados inicialmente 2.497 resumos de teses, dissertações e artigos nos bancos consultados, dos quais foram selecionados somente aqueles que abordassem especificamente a avaliação da inteligência. Dessa forma, foram selecionados 51 resumos do banco de teses da CAPES, 61 da base SCIELO, 61 da PEPSIC e 90 da PsycInfo, totalizando 263 trabalhos, os quais foram sistematizados de acordo com ano de publicação, origem (nacional ou internacional), tipo de pesquisa (empírica/teórica), modelos teóricos enfocados, amostra utilizada e instrumentos, de modo a, primeiramente, mapear essa produção em um período delimitando-a em termos de ano de publicação e, posteriormente, detalhar essa produção em termos de tendências, ênfases, escolhas metodológicas, teóricas e instrumentais.

\section{RESULTADOS E DISCUSSÃO}

O primeiro dado analisado visou localizar temporalmente a tendência das pesquisas na área, por meio do levantamento do número de trabalhos publicados por ano considerado. Optou-se por classificar os resultados de acordo com a base consultada, considerando-se também o número total de trabalhos analisados, cujos resultados podem ser visualizados na Figura 1. 


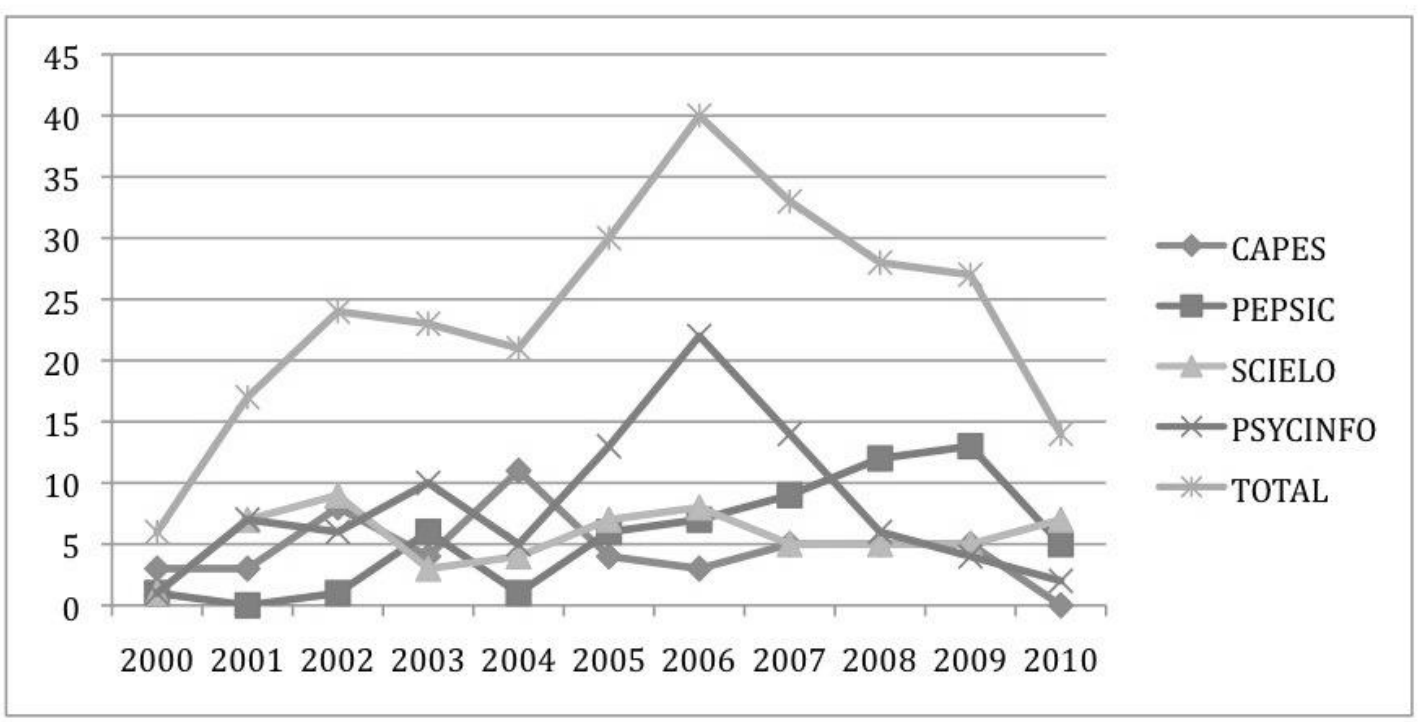

Figura 1. Produção científica por base de dado e ano de publicação.

De acordo com os dados obtidos, pode-se verificar que o pico no número de produções ocorre nos anos de 2005 e 2006, seguido de uma queda que se mantém nos anos seguintes. Ressalva deve ser feita, no entanto, ao fato de que a produção do ano de 2010 não contempla a produção da base de dados da Capes, visto que esta não se encontrava atualizada por ocasião da consulta.

Nota-se ainda que 263 trabalhos sobre avaliação da inteligência foram encontrados em um período de onze anos, uma média de 23,9 publicações por ano, um número que pode ser considerado baixo, se considerarmos o levantamento realizado por Primi (2003). Segundo o autor, tamanha a importância da inteligência para a Psicologia, que uma busca rápida na base de dados PsycInfo da Associação Americana de Psicologia (www.apa.org) "indicou que em pouco mais de um século existem mais de 18.400 artigos com a palavra inteligência em seu título" (p.68). Uma atualização desses dados foi realizada por Almeida, Guisande e Ferreira (2009), a qual permitiu aos autores afirmarem que a inteligência é provavelmente um dos assuntos mais estudados na Psicologia, visto que uma consulta em fevereiro de 2008 apontou a existência de 32.758 artigos. Mais especificamente focando a produção nacional, Primi e Flores-Mendoza (2006) verificaram a existência de 198 artigos publicados na base de dados Index-Psi, no período compreendido entre 1958 a 2000, os quais foram analisados pelos autores. Nota-se, dessa forma, que é grande o numero de publicações envolvendo o construto, embora um número bem menor considere a questão da sua avaliação.
Nesse sentido pode-se verificar que a ampliação no número de bases utilizadas na presente busca permitiu que esse quadro citado por estes autores fosse complementado, de modo que o número de trabalhos $(n=2.497)$ mostrou-se bem mais alto que o relatado por estes autores, ainda que uma ressalva deva ser feita em relação a uma possível limitação da pesquisa, visto que somente bases de dados eletrônicas foram consultadas, de forma que inúmeras publicações na área podem não ter sido encontradas, dado o fato da recenticidade dessas bases sob esse formato e da inserção das revistas nas mesmas. Dessa maneira acredita-se que esse valor pudesse ser ainda maior caso outras bases tivessem sido consultadas, bem como edições em formato não eletrônico e dentro de um período mais amplo, sendo esse tipo de estudo recomendado a fim de se traçar um panorama mais completo da área. Ainda que as pesquisas nacionais apresentem-se em pequeno numero se comparadas à literatura internacional, nesse subconjunto algumas tendências se fazem notar, as quais serão apontadas a seguir.

Foi possível notar, nos trabalhos investigados, uma predominância de pesquisas do tipo empírica $(n=188$, correspondendo a $71,5 \%$ da produção), principalmente aquelas que se constituem em relatos de pesquisa, embora outros tipos de estudos teóricos, tais como revisões de estado da arte, discussões teóricas e levantamentos bibliográficos, também tenham sido encontrados. É possível perceber que essa situação se repete em todos os bancos de dados analisados, em uma tendência contraria à situação encontrada por Primi e Flores-Mendoza (2006), segundo os quais a maior 
parte dos estudos tratava de reflexões teóricas sobre a temática $(35,4 \%)$, devendo-se considerar, entretanto, que a análise realizada pelos mesmos envolvia maior número de categorias nas quais as pesquisas poderiam ser enquadradas, não só teórica e empírica, como no presente estudo. Assim, se somadas as categorias consideradas empíricas (estudo correlacional, experimental, clínico/estudo de caso, propriedades psicométricas), teremos um valor de $47,9 \%$, de modo a se afirmar a predominância de estudos empíricos, assim como no estudo aqui relatado. Deve-se lembrar de que este artigo traz dados que revelam a predominância de trabalhos empíricos na área, embora estudos exploratórios sobre a história e os conceitos atuais sobre o construto também apresentam importante parcela de contribuição para o entendimento e compreensão sobre o tema na atualidade. A importância dessa diversidade de focos e métodos é ressaltada por Wagner e
Trentini, (2010), os quais ressaltam a importância de se estudar a inteligência por meio de diferentes formas.

$\mathrm{O}$ terceiro dado analisado referiu-se aos modelos teóricos utilizados nos resumos analisados. A importância desse tipo de levantamento ampara-se na constatação de que a definição desse construto depende, em grande parte, da orientação teórica adotada pelo pesquisador, conforme salientado por FloresMendoza, Nascimento e Castilho (2002), de modo que a presente análise teve por objetivo o conhecimento da concepção de inteligência subjacente às pesquisas analisadas. Dada a complexidade do fenômeno, Andrés-Pueyos (2006) salienta que não é de estranhar que a psicologia tenha abordado esse estudo a partir de muitos ângulos e modelos diferentes, dentre os quais se destacam o modelo psicométrico, o desenvolvimental, o cognitivo diferencial, sócio-cognitivo, abrangentes e globais, cuja sistematização foi utilizada no presente estudo.

Tabela 1

Modelos Teóricos Enfocados nas Pesquisas Analisadas

\begin{tabular}{|c|c|c|c|c|}
\hline Modelos de inteligência & Conceito-chave & Autores destacados & $\mathbf{F}$ & $\%$ \\
\hline Psicométrico & $\begin{array}{c}\text { Aptidões, capacidades, estruturas fatoriais e } \\
\text { hierárquicas }\end{array}$ & $\begin{array}{l}\text { Spearman, Thurstone, Cattell, } \\
\text { Guilford, Carrol, CHC }\end{array}$ & 136 & 51,71 \\
\hline Abrangentes & $\begin{array}{l}\text { Inteligências múltiplas, inteligência emocio- } \\
\text { nal }\end{array}$ & -- & 71 & 26,99 \\
\hline Desenvolvimental & Evolutivo & Piaget, Freud & 24 & 9,12 \\
\hline Cognitivo diferencial & Memória, atenção, etc & -- & 11 & 4,18 \\
\hline Globais & Conjunto de aptidões e habilidades & Sternberg, Gardner & 6 & 2,29 \\
\hline Sócio-cognitivo / culturais & Atributos sociais & Vygotsky & 2 & 0,76 \\
\hline Informação não fornecida & --- & --- & 13 & 4,95 \\
\hline TOTAL & & & 263 & 100 \\
\hline
\end{tabular}

De acordo com a Tabela 1, pode-se observar que a maioria dos estudos analisados é desenvolvida dentro do modelo psicométrico de inteligência (mais de $51 \%$ do total), cujos trabalhos envolvem o conhecimento acerca das aptidões, capacidades e como essas se estruturam em termos fatoriais ou hierarquicamente. Faz-se notar também uma quantidade importante de trabalhos desenvolvidos dentro de um modelo mais abrangente de inteligência (27\%), os quais envolvem conceitos como inteligências múltiplas ou inteligência emocional. Menos utilizados, mas ainda presentes podem ser encontrados estudos que fazem uso de modelos desenvolvimentais, cognitivos diferenciais, globais e sócio-cognitivos.
A partir de tais dados torna-se possível verificar que, nos últimos anos, os estudos que vêm sendo desenvolvidos na área têm se embasado nos modelos mais atuais e predominantes de inteligência, deixando de apresentar, cada vez mais, os focos tradicionais que caracterizaram, historicamente, a investigação da inteligência. Tal fato pode ser notado, por exemplo, diante da constatação de que os modelos desenvolvimentais, especificamente na perspectiva piagetiana, corresponderam a uma pequena parcela dos estudos mais atuais, de modo a apontar uma mudança no enfoque dos estudos ao longo dos anos. Isto porque, de acordo com os apontamento de Flores-Mendoza (2006), os mesmos correspondiam à maior parte $(67 \%)$ dos artigos sobre inteligência publicados nas décadas de 1970 e 1980 na revista Arquivos Brasileiros de Psicotécnica, 
assim como na revista Psicologia Teoria e Pesquisa entre os anos de 1985 a 1990.

O grupo dos modelos hierárquicos (os quais pressupõem dimensões mais gerais na parte superior da estrutura da inteligência e dimensões de menor generalidade, mais numerosas, na parte inferior), predominante nesse levantamento, é, segundo Flores-Mendoza e cols. (2002), o que mais tem contribuído para a prática da avaliação psicológica. Isso dado ao fato de que tem se mostrado mais vantajosa a utilização de testes de ampla generalidade do que um grande número de testes específicos durante a estimação da capacidade cognitiva de um indivíduo, aliado ao reconhecimento da teoria $\mathrm{CHC}$ como um dos principais avanços recentes na área, conforme salientado por Primi (2003).

Uma segunda tendência refere-se ao crescimento do número de estudos visando a investigação das inteligências múltiplas e da inteligência emocional (IE), a qual, embora relativamente recente, vem ganhando espaço dentro dessa temática (Almeida, Guisande \& Ferreira, 2009), dado o fato de que nenhuma das definições existentes de inteligência haviam, até o surgimento desse termo, feito referência explícita à associação entre a cognição e o uso de aspectos emocionais e sentimentais na cognição (Mayer, Salovey \& Caruso, 2008; Salovey \& Pizarro, 2004; Woyciekoski \& Hutz, 2009). Acompanhando os modelos mais atuais de inteligência, a IE também tem sido definida como um conjunto de habilidades ordenados hierarquicamente (MacCann \& Roberts, 2008), sendo alegado, por parte dos pesquisadores, sua capacidade de prever critérios clínicos, educacionais e ocupacionais além daqueles previstos pela inteligência geral (Zeidner, Roberts \& Matthews, 2008). Entretanto, convém salientar que o construto ainda tem sido alvo de discussões e controvérsias presentes na literatura internacional (Izard, 2001; Kaufman \& Kaufman, 2001; Mayer, Salovey, Caruso \& Sitarenios, 2001; Schaie, 2001).

Não se pode deixar de mencionar ainda a importância dos estudos com base nas teorias cognitivo diferencial, global e sócio-cognitiva, visto que tais modelos trazem contribuições importantes acerca do construto em seus diferentes focos, principalmente nos estudos voltados para a área educacional, clínica e organizacional.

Os achados vêm confirmar a percepção de Schelini (2006) de que a evolução das teorias de inteligência acabou por provocar uma mudança também nos focos dos estudos envolvendo a temática, assim como aumento do interesse pelo construto. Também sob essa ótica, Primi e Flores-Mendoza (2006) apontam que parece haver uma crescente maturidade nos estudos brasileiros no que se refere às abordagens utilizadas, acompanhando melhor o que ocorre no cenário internacional, ainda que, segundo os autores, se faça notar uma prevalência de discussões teóricas sobre as empíricas, o que, segundo Colom (2006), deve ser tratado como necessário, dada a importância de desenvolvimento de teorias psicológicas baseadas em fatores verificáveis empiricamente.

Dentre os estudos de avaliação da inteligência analisados, foi possível verificar que $41,4 \%$ deles fizeram menção ou uso de algum instrumento padronizado de medição do construto, enquanto os demais abordaram conceitos, teorias e/ou estudos sobre avaliação, fazendo uso de materiais próprios, muitas vezes não detalhados. Essa importante porcentagem reflete, na realidade, o número relativamente grande de testes psicológicos para avaliação da inteligência disponíveis no Brasil, situação apontada por Noronha, Primi e Alchieri (2004). De acordo com os autores, uma consulta aos 146 instrumentos de avaliação psicológica comercializados no país, na época, apontou que $41,1 \%$ desses eram específicos de inteligência e/ou aptidões. Dada essa diversidade, o levantamento dos instrumentais que foram utilizados nas publicações analisadas foi realizado, cujos resultados encontram-se disponíveis na Tabela 2.

A importância desse tipo de análise situa-se no fato de que várias investigações têm sido conduzidas, de acordo com Almeida, Primi e Ferreira (2008), procurando verificar em que medida as baterias de testes disponíveis, principalmente aquelas de largo espectro cognitivo, aproximam-se dos modelos teóricos mais recentes, tendo sido esse o objetivo do levantamento. 
Tabela 2

Instrumentos Utilizados nas Pesquisas

\begin{tabular}{ccccccc}
\hline Instrumentos & CAPES & PEPSIC & SCIELO & PSYCINFO & TOTAL F & $\%$ \\
\hline BPR-5 & 11 & 5 & 6 & 7 & 29 & 22,6 \\
WISC & 6 & 7 & 2 & 6 & 21 & 16,5 \\
MSCEIT & 0 & 7 & 4 & 10 & 21 & 16,5 \\
RAVEN & 6 & 6 & 6 & 3 & 21 & 16,5 \\
WAIS & 1 & 5 & 5 & 3 & 14 & 10,9 \\
DFH & 2 & 4 & 2 & 2 & 9 & 7,0 \\
R1 & 2 & 1 & 2 & 0 & 3 & 5,4 \\
WJ-II & 0 & 2 & 1 & 1 & 3 & 2,3 \\
Outros & 1 & 0 & 1 & $\mathbf{3 3}$ & $\mathbf{1 2 8}$ & $\mathbf{1 0 0 , 0}$ \\
\hline TOTAL & $\mathbf{2 9}$ & $\mathbf{3 7}$ & $\mathbf{2 9}$ & &
\end{tabular}

De acordo com a Tabela 2, pode-se observar que oito instrumentos para avaliação da inteligência foram utilizados nos trabalhos consultados. Deve-se, no entanto, esclarecer que os instrumentos citados referemse somente aos trabalhos que identificaram o instrumento no resumo, de modo que não foram considerados, nessa análise, os instrumentos que, eventualmente foram utilizados em outros trabalhos, mas não eram citados de modo específico no resumo. Dentre esses, destaque deve ser dado à Bateria de Provas de Raciocínio (BPR-5), utilizada em $22,6 \%$ dos estudos. Importante número de pesquisas também fez uso da Escala de Inteligência Wechsler para Crianças (WISC), Mayer Salovey Caruso Emotional Intelligence Test (MSCEIT) e o RAVEN (16,5\% cada). Dos instrumentos utilizados nas pesquisas, salienta-se que dois ainda não se encontram publicados em nosso país (MSCEIT e Woodcock-Johnsson III), sendo o primeiro específico para avaliação da inteligência emocional e o outro para avaliação da inteligência dentro do modelo CHC.

Alguns dos instrumentos utilizados nacionalmente são os mesmos apontados por Flanagan e Harrison (2005), que destacam, dentro do panorama internacional, o Wechsler Intelligence Scale for Children-WISCIII, Wechsler Adult Intelligence Scale-WAIS-III e a Bateria de Habilidades Cognitivas Woodcock-Johnson III como os mais utilizados na avaliação da inteligência. Entretanto, torna-se importante salientar que o Brasil conta hoje, com 18 outros instrumentais para avaliação desse construto, aprovados pelo Conselho Federal de Psicologia (www.pol.Ed.br/satepsi), sendo que somente onze foram utilizados nas pesquisas consultadas. Note-se que outros testes também foram encontrados nos resumos analisados, no entanto, por não representarem porcentagem significativa total (inferior a 1\%), compuseram a linha referente a "outros" na Tabela. São eles: Teste não verbal de inteligência G36, Teste de inteligência não verbal (TONI3) e Teste não verbal de inteligência R2.

Tais resultados chamam a atenção para o fato de que, ainda que a teoria $\mathrm{CHC}$ venha sendo usada, gradualmente, como nomenclatura padrão entre profissionais e pesquisadores no entendimento da inteligência, conforme apontado por Primi (2003), nenhuma das baterias disponíveis no mercado apresenta uma avaliação completa das dez capacidades amplas englobadas neste modelo. Como exemplo, o autor cita o WISC-III, cuja composição reflete principalmente três fatores amplos (inteligência cristalizada, processamento visual e velocidade de processamento), tendo sido criticada por não avaliar áreas importantes como a inteligência fluida, capacidade de armazenamento e recuperação da memória a longo prazo e processamento auditivo, devendo-se citar ainda que tal bateria apresenta um número maior do que o necessário de subtestes em algumas áreas, tais como processamento visual (composta por quatro subtestes), cujos problemas ainda permanecem na nova versão do instrumento (WISC-IV), de modo que torna-se necessário investir no desenvolvimento e/ou adaptação de novas provas derivadas diretamente do modelo $\mathrm{CHC}$.

Nesse sentido, buscando sanar essa lacuna existente, os estudos que vêm sendo conduzidos no sentido de adaptação da Bateria de Habilidades Cognitivas Woodcock-Johnson III tem recebido destaque no Brasil por ser este um dos poucos testes disponíveis embasados no modelo mais atual de inteligência, o CHC (Chiodi \& Wechsler, 2009). Disponível em duas versões, uma direcionada para avaliação das habilidades cognitivas (forma padrão) e uma segunda versão (am- 
pliada) voltada à avaliação do rendimento acadêmico (Mather \& Gregg, 2002; Sandoval \& Woodcock, 2005), tal bateria tem se constituído no instrumento mais completo para explicação do funcionamento intelectual. Estudos brasileiros com o instrumento foram realizados visando a construção de uma versão brasileira da forma padrão (Wechsler \& Schellini, 2006; Wechsler, Vendramini \& Schellini, 2007), bem como sua forma ampliada (Chiodi, 2012). Entretanto deve-se ressaltar que a mesma ainda não se encontra disponível comercialmente.

Assim o que se pode verificar é que um dos problemas dos testes de inteligência que vêm sendo utilizados no país refere-se à dificuldade dos mesmos em representar a multiplicidade de aspectos que intervêm na cognição humana (Almeida, Primi \& Ferreira, 2008), ainda que Flores-Mendoza (2006) aponte para o fato de que, particularmente para a psicologia no Brasil, o início do terceiro milênio caracterize-se pelo esforço técnico-científico de profissionais e pesquisadores na tentativa de melhora dos instrumentos psicológicos já existentes, assim como a criação de outras medidas.

Atenção deve ser dada ao fato de que grande parte dos instrumentos disponíveis atualmente é relativamente recente, visto que levantamento realizado por Primi (2003) apontou uma escassez de instrumentos para avaliar a inteligência, a qual também foi ressaltada por Weschler, Vendramini e Schelini (2007) ao enfatizarem a importância de adaptações de materiais internacionais como forma de suprir essa lacuna. Diante desta percepção, a necessidade de investigações deve ser ressaltada, principalmente aquelas visando a criação, adaptação e validação de instrumentos para a população brasileira.Isso porque a importância da avaliação da inteligência ampara-se na constatação de que a observação das capacidades diferenciadas pode permitir a elaboração de um diagnóstico não só das deficiências cognitivas, mas também do alto desempenho, bem como a análise de diferenças individuais, tendo em vista as decisões ao nível do tratamento educativo diferenciado (Almeida, 2002).

$\mathrm{O}$ terceiro dado analisado referiu-se à amostra que vem sendo enfocada nas investigações sobre avaliação da inteligência. A fim de facilitar a classificação dos dados, os participantes foram divididos em faixas de desenvolvimento (crianças, adolescentes, jovens, adultos e idosos), conforme pode ser visualizado na Tabela 3. Salienta-se que o número total de trabalhos utilizados nessa análise ultrapassa a quantidade de trabalhos empíricos considerados, dado o fato de que alguns estudos fizeram uso de mais de um tipo de amostra, tendo sido consideradas todas as ocorrências.

Tabela 3

Amostras Utilizadas nas Pesquisas Analisadas

\begin{tabular}{|c|c|c|c|c|c|c|}
\hline Faixa de desenvolvimento & CAPES & PEPSIC & SCIELO & PSYCINFO & TOTAL F & $\%$ \\
\hline $\begin{array}{l}\text { Crianças } \\
\text { (0 a } 12 \text { anos })\end{array}$ & 10 & 17 & 13 & 14 & 54 & 22,0 \\
\hline $\begin{array}{l}\text { Adolescentes } \\
\text { (13 a } 18 \text { anos) }\end{array}$ & 9 & 15 & 12 & 15 & 51 & 20,8 \\
\hline $\begin{array}{c}\text { Jovens } \\
\text { (19 a } 25 \text { anos) }\end{array}$ & 14 & 15 & 14 & 25 & 68 & 27,8 \\
\hline $\begin{array}{c}\text { Adultos } \\
\text { (26 a } 59 \text { anos) }\end{array}$ & 9 & 13 & 11 & 22 & 55 & 22,4 \\
\hline $\begin{array}{c}\text { Idosos } \\
\text { (igual ou acima de } 60 \text { anos) }\end{array}$ & 6 & 3 & 4 & 4 & 17 & 6,9 \\
\hline Total & 48 & 63 & 54 & 80 & 245 & 100,0 \\
\hline
\end{tabular}

A partir da Tabela 3 pode-se observar, de uma forma geral, um equilíbrio entre o número de pesquisas desenvolvidas em cada faixa de desenvolvimento considerada, com exceção dos estudos desenvolvidos com idosos, os quais aparecem em somente 6,9\% dos trabalhos. Pode-se notar uma ligeira predominância de estudos investigando a inteligência na população jovem (cerca de $28 \%$ ), voltados principalmente ao estu- do de universitários e educadores. Tal dado difere, em partes, da percepção de Almeida, Lemos, Guisande e Primi (2008), segundo os quais, a identificação dos fatores explicativos da habilidade intelectual e do desempenho cognitivo ao longo da infância e adolescência tem sido um dos alvos privilegiados da psicometria, ainda que, no presente estudo, crianças e adolescentes também tenham sido foco de importante 
número de estudos. Por outro lado, a ausência de materiais científicos publicados sobre o tema em relação aos idosos também é apontada por Rozestraten (2002) em seu trabalho sobre a evolução dos estudos da inteligência nesta população.

O interesse na diferenciação das habilidades cognitivas entre crianças e adultos se tem feito presente entre os pesquisadores (Guilford, 1966), de modo que a estimação da influência da idade sobre o desenvolvimento do desempenho intelectual tem se mostrado um problema crucial na pesquisa educacional, apresentando, segundo Cliffordson e Gustafsson (2008), consideráveis implicações teóricas, metodológicas e práticas. Por esse motivo, a literatura tem destacado o impacto da idade sobre as diferentes aptidões cognitivas (Almeida, 1988), de modo que uma leitura dos resultados combinados da Tabela 2 (instrumentos) e Tabela 3 (amostras por faixa etária) será feita a seguir.

Uma análise do público a que se destinam os instrumentos mais utilizados (constantes na Tabela 2) mostra que os testes BPR-5, MSCEIT, WJ-II, R1 e WAIS são direcionados ao público jovem e adulto. Neste sentido, se considerarmos a predominância da utilização de jovens como principal amostra dos estudos, conforme apontado na Tabela 3 tornar-se-á compreensível o fato da BPR-5 ter se destacado entre os instrumentais encontrados visto que autores como Godoy, Noronha, Ambiel e Nunes (2008) e Souza, Primi e Miguel (2007), apontam-na como sendo o instrumento mais utilizado para a população jovem e adulta, uma vez que seu uso encontra-se bastante direcionado à orientação profissional e seleção pessoal, sendo também comumente empregado como medida de aptidões e raciocínio geral, por meio da avaliação de cinco áreas (verbal, numérica, abstrata, mecânica e espacial). Um desses instrumentos (R1) avalia somente a capacidade geral, ao passo que o MSCEIT contempla um tipo específico de inteligência, a emocional, devendo-se ressaltar que o mesmo não se encontra publicado em nosso país, ainda que vários estudos venham sendo conduzidos (Cobêro, Primi \& Muniz, 2006; Dantas \& Noronha, 2005; Freitas \& Noronha, 2006; Miguel \& Noronha, 2009; Muniz \& Primi, 2007; Muniz, Primi \& Miguel, 2007; Noronha \& Jesus Jr., 2008; Primi, Bueno \& Muniz, 2006).

A análise permitiu verificar a utilização de somente dois instrumentos voltados para a avaliação de crianças, o WISC-III e o DFH. O primeiro deles constitui-se, de acordo com Yales e cols. (2006), nas baterias de avaliação de QI mais conhecidas e utilizadas em nosso meio, ainda que autores como Almeida, Primi e
Ferreira (2008) apontem o fraco suporte teórico dessas escalas visto que, segundo tais autores, pesquisas demonstraram que o WAIS-III cobre seis domínios de funcionamento cognitivo (compreensão verbal, raciocínio perceptivo, velocidade de processamento, memória de trabalho, memória auditiva e memória visual), de modo a se mostrarem consistentes com as atuais teorias da inteligência, nomeadamente o modelo $\mathrm{CHC}$, ainda que não avalie todas as dimensões do mesmo. Entretanto, a mesma situação não ocorre em relação ao WISC-IV, cujos estudos mais recentes sugeriram uma inadequação desta bateria a esse modelo teórico.

Por outro lado, o DFH tem sido bastante utilizado em amostras infantis, embora não seja descartada sua aplicação em adultos (Bandeira, Costa \& Arteche, 2007). De acordo com Rueda, Bartholomeu e Sisto (2006), tem se mostrado uma das técnicas psicológicas mais utilizadas, frequentemente para medir a inteligência de crianças, tendo sido empregado na investigação das dificuldades de aprendizagem, desenvolvimento cognitivo, criatividade, autoestima e depressão, de forma que "sua avaliação tem sido, principalmente, dedicada às finalidades clínicas" (Wechsler \& Schelini, 2002, p.31). Ainda de acordo com as autoras, seu uso é recomendado sempre em combinação com outras medidas de funcionamento intelectual, visto o fato de o instrumento avaliar somente aspectos não verbais do funcionamento cognitivo infantil, de forma a não abranger várias outras facetas que compõem o conceito de inteligência. Uma última observação fica por conta do Raven, o qual ainda que comumente mais utilizado com crianças de 6 a 12 anos, também pode ser aplicado em outras faixas etárias.

\section{CONSIDERAÇÕES FINAIS}

O presente trabalho pretendeu explorar as tendências existentes no estudo da avaliação da inteligência, considerando-se as investigações que foram publicadas em quatro importantes bases de dados nos últimos onze anos. A partir dessa análise foi possível observar que o interesse nos estudos de inteligência tem aumentado, de maneira a demonstrar a importância da sua avaliação nas mais diversas áreas da Psicologia.

A identificação dos aspectos relevantes da produção científica brasileira sobre avaliação da inteligência apontou para um quadro marcado por tendências que acompanham o cenário internacional, visto que a maior parte dos estudos tem embasando-se nos modelos teóricos mais atuais. Pode ser verificado também o 
emprego de diferentes instrumentos para avaliação do construto, sendo que, dentre esses, foram encontrados instrumentos tradicionalmente utilizados em nosso país e outros ainda em fase de estudo e validação, de modo a apontar para o fato de que o interesse na temática ainda se mantém voltado à identificação da inteligência de indivíduos das mais variadas faixas etárias.

Espera-se que os resultados apontados por esse trabalho possam contribuir para a expansão dos estudos sobre a temática ao clarificar os enfoques que têm sido dados nos estudos, por meio da identificação das contribuições que se tem alcançado através dos estudos brasileiros, bem como as necessidades e discussões que ainda se fazem presentes. Almeja-se que este trabalho possa inspirar outros pesquisadores, contribuindo para a expansão do conhecimento acerca da inteligência na população brasileira.

\section{REFERÊNCIAS}

Obs.: Referências precedidas de um asterisco indicam estudos incluídos na revisão.

Ackerman, P. L., \& Beier, M. E. (2005). Knowledge and intelligence. In O. Wilhelm \& R. W. Engle (Eds.). Handbook of understanding and measuring intelligence (pp.125-139). Thousand Oaks, CA: Sage.

Almeida, L. S. (1988). O impacto das experiências educativas na diferenciação cognitiva dos alunos: Análise dos resultados em provas de raciocínio diferencial. Peritia: Revista Portuguesa de Psicologia, 24, 131-157.

*Almeida, L. S. (2002). As aptidões na definição e avaliação da inteligência: O concurso da análise fatorial. Paideia, 12, 5-17.

Almeida, F. (2009). Teste das Matrizes Progressivas de Raven. Peritia: Revista Portuguesa de Psicologia. Retirado de http://www.revistaperitia.org/wpcontent/uploads/2010/04/MPCR.pdf

Almeida, L., Guisande, M. A., \& Ferreira, A. (2009). Inteligência: Perspectivas teóricas. Coimbra: Almedina.

Almeida, L., Guisande, M. A., Primi, R., \& Ferreira, A. (2008). Construto e medida da inteligência: Contributos da abordagem factorial. In A. Candeias, L. S. Almeida, A. Roazzi \& R. Primi (Eds.), Inteligência: Definição e medida na confluência de múltiplas concepções (pp. 49-80). São Paulo: Casa do Psicólogo.

*Almeida, L. S., Lemos, P. G., Guisande, P. M. A., \& Primi, R. (2008). Inteligência, escolarização e idade: Normas por idade ou série escolar?. Avaliação Psicológica, 7, 117-125.

Almeida, L. S., \& Primi, R. (2009). Considerações em torno da medida de inteligência. In L. Pasquali (Ed.), Instrumentação psicológica (pp. 387-410). Porto Alegre: Artmed.

Alves, I. C. B. (1998). Variáveis significativas na avaliação da inteligência. Psicologia Escolar e Educacional , 2, 109-114.

Andrés-Pueyo, A. (2006). Modelos psicométricos da inteligência. In C. E. Flores-Mendoza \& R. Colom (Eds.), Introdução à psicologia das diferenças individuais (pp. 73-100). Porto Alegre: Artmed.

Armstrong, T. (2003). Muitos tipos de inteligência: A teoria das inteligências múltiplas. In T. Armstrong (Ed.), 7 tipos de inteligência (O. Júnior, Trans.) (pp. 17-23). Rio de Janeiro: Record.
*Bandeira, D. R., Costa, A., \& Arteche, A. (2007). Estudo de validade do DFH como medida de desenvolvimento cognitivo infantil. Psicologia: Reflexão e Critica, 21, 332-337.

Brodi, N. (2000). History of theories and measurement of intelligence. In R. J. Sternberg (Ed.), Handbook of intelligence (pp. 16-33). Cambridge, MA: Cambridge University Press.

Carroll, J. B. (1997). The three-stratum theory of cognitive abilities. In D. P. Flanagan, J. L. Genshaft \& P. L. Harrison (Eds.), Contemporary intellectual assessment: Theories, tests and issues (pp. 122-130). New York, NY: Guilford Press.

Chen, J., \& Gardner, H. (1997). Alternative assessment from a multiple intelligences: Theoretical perspective. In D. P. Flanagan, J. L. Genshaft \& P. L. Harrison (Eds.), Contemporary intellectual assessment: Theories, tests and issues (pp. 105-121). New York, NY: Guilford Press.

*Chiodi, M. G., \& Wechsler, S. M. (2009). Escala de Inteligência WISC-III e Bateria de Habilidades Cognitivas Woodcock Johnson-III: Comparação de instrumentos. Avaliação Psicológica, 8, 313-324.

Cliffordson, C., \& Gustafsson, J. (2008). Effects of age and schooling on intellectual performance: Estimates obtained from analysis of continuous variation in age and length of schooling. Intelligence, 36, 143-152.

Colom, R. (2006). História da psicologia das diferenças individuais. In C.E. Flores-Mendoza \& R. Colom (Eds.), Introdução à psicologia das diferenças individuais (pp. 15-36). Porto Alegre: Artmed.

Coutinho, A. C. A. M., \& Nascimento, E. (2010). Formas abreviadas do WAIS-III para avaliação da inteligência. Avaliação Psicológica, 9, 25-33.

Davidson, J. E., \& Downing, C. L. (2000). Contemporary models of intelligence. In R. J. Sternberg (Ed.). Handbook of intelligence (pp. 34-49). Cambridge, MA: Cambridge University Press.

*Figueiredo, V. L. M., \& Pinheiro, S. (1998). O teste WISC-III em uma amostra do Rio Grande do Sul. Temas em Psicologia, 6, 255-261.

Flanagan, D. P., McGrew, K. S., \& Ortiz, S. O. (2000). The Wechsler Intelligence Scales and gf-gc theory. Boston, MA: Allyn and Bacon.

Flanagan, D. P., \& Harrison, P. L. (2005). Contemporary intellectual assessment. New York, NY: Guilford Press.

Flores-Mendoza, C. E. (2006). O estudo das diferenças individuais no Brasil. In C.E. Flores-Mendoza \& R. Colom (Eds.), Introdução à psicologia das diferenças individuais (pp. 37-56). Porto Alegre: Artmed.

*Flores-Mendoza, C. E., Nascimento, E., \& Castilho, A. V. (2002). A crítica desinformada aos testes de inteligência. Estudos de Psicologia (Campinas), 19, 17-36.

Gaspari, J. C. \& Schwarts, G. M. (2002). Inteligências múltiplas e representações. Psicologia: Teoria e Pesquisa, 18, 261-266.

*Godoy, S., Noronha, A. P .P., Ambiel, R. A. M., \& Nunes, M. F. O. (2008). Instrumentos de inteligência e interesses em orientação profissional. Estudos de Psicologia, 13, 75-81.

Gottsfritz, M. O., \& Alves, I. C. B. (2009). Normas do Teste de Inteligência Não Verbal R-1 para adultos não alfabetizados. Interação em Psicologia, 13, 59-68.

Guilford, J. P. (1966). Intelligence: 1965 model. American Psychologist, 21, 20-26.

Horn, J. L., \& Noll, J. (1997). Human cognitive capabilities: GFGc theory. In D. P. Flanagan, J. L. Genshaft \& P. L. Harrison (Eds.), Contemporary intellectual assessment: Theories, tests and issues (pp. 53-91). New York, NY: Guilford Press. 
Ittenbach, R. E., Esters, I. G., \& Wainer, H. (1997). The history of test development. In D. P. Flanagan, J. L. Genshaft \& P. L. Harrison (Eds.), Contemporary intellectual assessment: Theories, tests and issues (pp. 17-31). New York, NY: Guilford Press.

Izard, C. E. (2001). Emotional intelligence or adaptive emotions?. Emotion, 1, 249-257.

Kaufman, A. S., \& Kaufman, J. C. (2001). Emotional intelligence as an aspect of general inteligence: What would David Wechsler say?. Emotion, 1, 258-264.

MacCann, C., \& Roberts, R. D. (2008). New paradigms for assessing emotional intelligence: Theory and data. Emotion, 8, 540-551.

Mather, N., \& Gregg, N. (2002). Assessment with the Woodcock-Johnsson III. Washington, DC: Psychological Corporation.

Mayer, J.D., Salovey, P. \& Caruso, D.R. (2008). Emotional intelligence: new ability or ecletic traits?. American Psychologist, 63, 503-517.

Mayer, J. D., Salovey, P., Caruso, D. R., \& Sitarenios, G. (2001). Emotional intelligence as a standard intelligence. Emotion, 1, 232-242.

*Noronha, A.P . P., \& Jesus Júnior, A. G. (2008). Parâmetros psicométricos do Mayer Salovey Caruso Emotional Intelligence Test - MSCEIT. Psic, 9, 145-153.

*Noronha, A. P. P., Primi, R., \& Alchieri, J. C. (2004). Parâmetros psicométricos: Análises dos testes psicológicos comercializados no Brasil. Psicologia: Ciência e Profissão, 24, 88-99.

Nascimento, E. (2004). Adaptação, validação e normatização de uma amostra brasileira. In E. Nascimento (Ed.), WAIS-III: Escala de Inteligência Wechsler para Adultos - Manual para administração e avaliação (pp. 161-192). São Paulo: Casa do Psicólogo.

*Primi, R. (2002). Inteligência fluida: Definição fatorial, cognitiva e neuropsicológica. Paideia, 12, 57-75.

* Primi, R. (2003). Inteligência: Avanços nos modelos teóricos e nos instrumentos de medida. Avaliação Psicológica, 1, 67-77.

Primi, R., \& Flores-Mendoza, C. E. (2006). O estudo da inteligência no Brasil. In C. E. Flores-Mendoza \& R. Colom (Eds.), Introdução à psicologia das diferenças individuais (pp. 189200). Porto Alegre: Artmed.

*Primi, R., Santos, A. A. A., Vendramini, C. M., Muller, F. T. F. A., Lukjanenko, M. F., \& Sampaio, I. S. (2001). Competências e habilidades cognitivas: Diferentes definições dos mesmos construtos. Psicologia: Teoria e Pesquisa, 17, 151-159.

*Roazzi, A., \& Souza, B. C. (2002). Repensando a inteligência. Paideia, 12, 31-55.

Roberts, R. D., Markham, P. M., Matthews, G., \& Zeider, M. (2005). Assessing intelligence: Past, present and future. In O. Wilhelm \& R. W. Engle, Handbook of understanding and measuring intelligence (pp. 333-360). Thousand Oaks, CA: Sage.

*Rozestraten, R. J. A. (2002). Evolução inicial dos estudos da inteligência do idoso: Mitos e fatos. Paideia, 12, 149-162.

*Rueda, F. J. M., Bartholomeu, D., \& Sisto, F. F. (2006). Maturidade perceptual e inteligência. Psicologia: Ciência e Profissão, $26,490-503$.
*Santos, A. A. A., Noronha, A. P. P., \& Sisto, F. F. (2005). Teste de Inteligência R1-Forma B G36: Evidência de validade convergente. Estudos de Psicologia (Natal), 10, 191-197.

Schaie, K. W. (2001). Emotional intelligence: Psychometric status and developmental characteristics. Emotion, 1, 243-248.

*Schelini, P. W. (2006). Teoria das inteligências fluida e cristalizada: Início e evolução. Estudos de Psicologia (Natal), 11, 323-332.

Schulze, R. (2005). Modeling structures of intelligence. In O. Wilhelm \& R.W. Engle (Eds.). Handbook of understanding and measuring intelligence (pp. 241-263). Thousand Oaks, Califórnia: Sage Publications.

*Sisto, F. F., Ferreira, A., \& Matos, M. P. B. (2006). TCR e R1: Duas medidas do fator g. Psicologia em Estudo, 7, 69-77.

*Sisto, F. F., Santos, A. A. A., \& Noronha, A. P. P. (2007). Estudo sobre a dimensionalidade do teste R1 - Forma B. Psicologia em Estudo, 12, 185-193.

*Souza, C. V. R., Primi, R., \& Miguel, F. K. (2007). Validade do Teste Wartegg: Correlação com 16PF, BPR-5 e desempenho profissional. Avaliação Psicológica, 6, 39-49.

Thorndike, R. M. (1997). The early history of intelligence testing. In D. P. Flanagan, J. L. Genshaft, \& P. L. Harrison (Eds.), Contemporary intellectual assessment: Theories, tests and issues (pp. 3-16). New York, NY: Guilford Press.

Urbina, S. (2004). Essentials of psychological testing. Hoboken, NJ: John Wiley \& Sons.

Wagner, F., \& Trentini, C. M. (2010). Estratégias de avaliação rápida da inteligência através das Escalas Wechsler. Revista Neuropsicologia Latinoamericana, 2, 47-54.

Wechsler, S. M. (não publicado). Bateria de Avaliação da Inteligência para Crianças.

*Wechsler, S. M., \& Schelini, P. W. (2006). Bateria de habilidades cognitivas Woodcock-Johnson III: Validade de construto. Psicologia: Teoria e Pesquisa, 22, 287-296.

*Wechsler, S. M., \& Schelini, P. W. (2002). Validade do Desenho da Figura Humana para avaliação cognitiva infantil. Avaliação Psicológica, 1, 29-38.

*Wechsler, S. M., Vendramini, C. M. M. \& Schelini, P. W. (2007). Adaptação brasileira dos testes verbais da bateria Woodcock-Johnson III. Interamerican Journal of Psychology, 41, 285-294.

Wilhelm, O., \& Engle, R. W. (2005). Intelligence: A diva and a workhorse. In O. Wilhelm \& R.W. Engle (Eds.), Handbook of understanding and measuring intelligence (pp. 1-10). Thousand Oaks, CA: Sage.

Zeidner, M., Roberts, R. D., \& Matthews, G. (2008). The science of emotional intelligence: Current consensus and controversies. European Psychologist, 13, 64-78.

Recebido: 18/08/2011

Última revisão: 17/05/2012 Aceito: 03/07/2012 